\title{
Five years review of extraction frequencies at S.D.M. College of dental sciences and Hospital in orthodontic department
}

\author{
Shanthiprasad Indra ${ }^{1}$, G. Arun-Kumar ${ }^{2}$, Niranjanaprasad Indra ${ }^{3}$, GC. Ramesh ${ }^{4}$, Ganesh Chinthan ${ }^{5}$, Sachin \\ Bharadwaj $^{6}$
}

${ }^{1}$ Senior Lecturer, Department of Orthodontics \& Dentofacial Orthopedics, Sharavathi Dental College \& Hospital, Shivamogga, India

${ }^{2}$ Professor and Head of the Department, Department of Orthodontics \& Dentofacial Orthopedics, Sharavathi Dental College \& Hospital, Shivamogga, India

${ }^{3}$ Associate Professor, Department of Oral and Maxillofacial Surgery, Institute of Dental Sciences, Bareilly, Uttar Pradesh, India

${ }^{4}$ Professor, Department of Orthodontics \& Dentofacial Orthopedics, Sharavathi Dental College \& Hospital, Shivamogga, India

${ }^{5}$ Associate Professor, Department of Orthodontics \& Dentofacial Orthopedics, Sharavathi Dental College \& Hospital, Shivamogga, India

${ }^{6}$ III Year Post-Graduate Student, Department of Orthodontics and Dentofacial Orthopaedics, Sharavathi Dental College and Hospital, Shivamogga, Karnataka, India

Correspondence:

Department Of Orthodontics \& Dentofacial Orthopedics Sharavathi Dental College \& Hospital, Shivamogga, India yesprasad11@gmail.com

Indra S, Arun-Kumar G, Indra N, Ramesh GC, Chinthan G, Sachin Bharadwaj. Five years review of extraction frequencies at S.D.M. College of dental sciences and Hospital in orthodontic department. J Clin Exp Dent. 2019;11(11):e991-9.

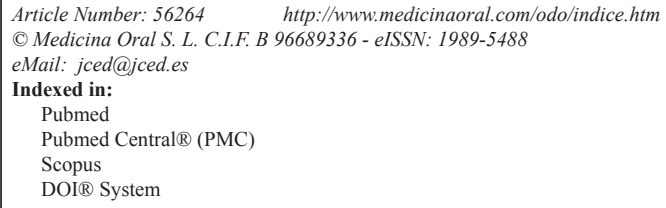

\begin{abstract}
Background: To find out the frequency of extraction in general, in Class I, Class II Class III patients, and to compare the frequency of extraction among sex and age.

Material and Methods: 550 cases were selected retrospectively having detailed case history, complete records of facial photographs, lateral cephalogram, orthopantomographs and study models. Frequency of extraction was evaluated separately for class I, class II and class III malocclusion and for sex and ages, using the records collected. Results: Show that there was $59.80 \%$ of extraction in general. Comparison of sex shows that there were 66.60 of extraction in females. The mean age of males for extraction was $17.85+/-4.18$ and the mean age of females was $18.36+/ 4.89$. Among all the groups, Class I malocclusion shows $89 \%$ of extraction.

Conclusions: There was higher frequency of extraction comprising in general. Comparison of sex shows that there was higher frequency of extraction in females. Comparison of age shows that extraction frequency is more in late adolescent period. Among all the groups, Class I malocclusion shows higher frequency of extraction.
\end{abstract}

Key words: Extraction, frequency, malocclusion.raumatic neuroma; palisaded encapsulated neuroma; oral palisaded encapsulated neuroma. 


\section{Introduction}

For more than 100 years, soon after that the practitioners recognized that orthodontic treatment can influence the patients' profile and esthetics, the extraction of teeth in orthodontics has been a matter of debate (1). In the early 20th century, Edward Angle and his followers believed that extraction destroyed the possibility of ideal occlusion or esthetics (2). As it become clear that arches could and did collapse after expansion despite efforts to produce ideal function, extraction was reintroduced in 1930s in an attempt to overcome relapse problems. By mid-century, extraction had become common place among orthodontists using tweeds modification of edgewise appliance. Tweed has advocated the extraction of 4 premolars to attain facial esthetics and denture similar to those in non orthodontic normal's (3). To attain this tweed advocated that the mandibular incisor in relation to the basal bone should be $90+/-5$ degrees. The Begg technique was introduced in Australia and many orthodontists who had not used edgewise adopted the Begg approach and began to extract more frequently and the percentage of orthodontic patients with extraction reached a peak (2). Orthodontic treatment by removing teeth had been widely accepted for many types of patients for better long term stability, but non-extraction treatment have again gained widespread popularity with the concern of condylar displacement, narrowed smiles with dark corners, and dished-in profiles with extraction $(4,5)$. Since then extraction percentages have declined noticeably. Extraction frequency is used as a statistical measure describing the number of orthodontic patients having permanent tooth extraction, and it is expressed as a percentage of total treatment samples. It is an unemotional statistic reflecting the sum of all the variables associated with the extraction question. Sometimes, including premolar extraction, produce changes in the facial profile. Therefore, it is useful for the clinical to know the efforts of different treatment options and what they offer to the patients.

-Aims and Objectives

1. To find out the frequency of extraction in S.D.M. College of Dental Sciences and Hospital, Dharwad. for 5 years from 2007 to 2012.

2. To find out the frequency of extraction in Class I, Class II Class III patients.

3. To find out frequency of extraction among age and sex.

\section{Material and Methods}

The records for this investigation were drawn retrospectively over a period of five years from S.D.M. College of Dental Sciences and Hospital Sattur, Dharwad, Karnataka, India from year 2007 to 2012.

The records involved pretreatment study models and pretraced lateral cephalograms which were traced by the respective postgraduate to whom the case was allotted. The treatment plan was decided by the same head of the department for all the five years.

Case selection was based on the following criteria:

1. Patients without any history of orthodontic treatment

2. Age range between $10-23$ years

3 . None of the cases had congenital and dentofacial anomalies or significant facial asymmetries

4. Cases involving surgical treatment were excluded.

-Subject and Methods

Based on inclusion criteria a total of 550 cases were selected having complete records. For all the cases a detailed case history was taken along with facial photographs lateral cephalograms, orthopantamographs, and study models. All cephalograms were obtained on the same cephalometric unit [PMHFCC proline with a cephalostat, manufactured by planmaca OY, Helsinki, FINLAND, with the same magnification of 1:1.09]. The cassette used was Kodak lanex - Omatic, USA.

Table 1: Frequency of extraction in each group.

\begin{tabular}{|l|c|c|c|c|c|}
\hline & Class & & Extraction & Nonextraction & Total \\
\hline GR & & Count & 181 & 122 & 303 \\
\hline & 1 & $\%$ Within GR & $59.7 \%$ & $40.3 \%$ & $100.0 \%$ \\
\hline & & $\%$ Within EXTNTS & $55.0 \%$ & $55.2 \%$ & $55.1 \%$ \\
\hline & 2 & Count & 142 & 89 & 231 \\
\hline & & $\%$ Within GR & $61.5 \%$ & $38.5 \%$ & $100 \%$ \\
\hline & & $\%$ Within EXTNTS & $43.2 \%$ & $40.3 \%$ & $42.0 \%$ \\
\hline & 3 & Count & 6 & 10 & 16 \\
\hline & & $\%$ Within GR & $37.5 \%$ & $62.5 \%$ & $100 \%$ \\
\hline & & $\%$ Within EXTNTS & $1.8 \%$ & $4.5 \%$ & $2.9 \%$ \\
\hline & & Count & 329 & 221 & 550 \\
\hline & & $\%$ Within GR & $59.8 \%$ & $40.2 \%$ & $100 \%$ \\
\hline
\end{tabular}


All cephalograms were hand traced by the respective postgraduate on an acetate mattracing paper with $2 \mathrm{H}$ LEAD PENSIL. The following cephalometric analysis:

From the case history files the age sex and malocclusion group, to which the patient belongs to was recorded. All these values were transferred from the files of the each to the extraction table or nonextraction table (Table 1). The age and sex of the patient were compared among extraction and nonextraction tables and two main variables were further classified as class I class II class III groups, which were further subdivided into extraction and nonextraction subgroups. So a total of 6 tables were obtained. They were the class I extraction, class II non extraction, class II extraction, class II non extraction, class III extraction, class III non extraction subgroups.

-Statistical Analysis

Statistical analysis was done using the SSPS software [SPSS for windows XP version 13, SSPS inc, Chicago]. First the independent test was done to compare the sub- groups within extraction and non extraction. Then compared among age sex and the 3 malocclusions groups with a multiple comparison bonferroni test to compare among subgroups.

\section{Results}

In this study, the total number of subjects was 55 which comprised of 209 [38\%] males and 341 females (Fig. 1) they were divided into 3 subgroups according to angles classification as class I class II, class III malocclusion (Fig. 2).

The overall frequency of extraction from the 550 subjects was 329 [59\%] and the number of subjects who underwent non extraction was $221\{40.2 \%]$ (Fig. 3).

The mean age of males was $17.85=/-4.18$ and the mean age of females was $18.56+/-4.89$ which was not statistically significant [males $p=.192$, females $p=.206$ ] (Fig. 4).

When relating sex with the frequency of extraction for

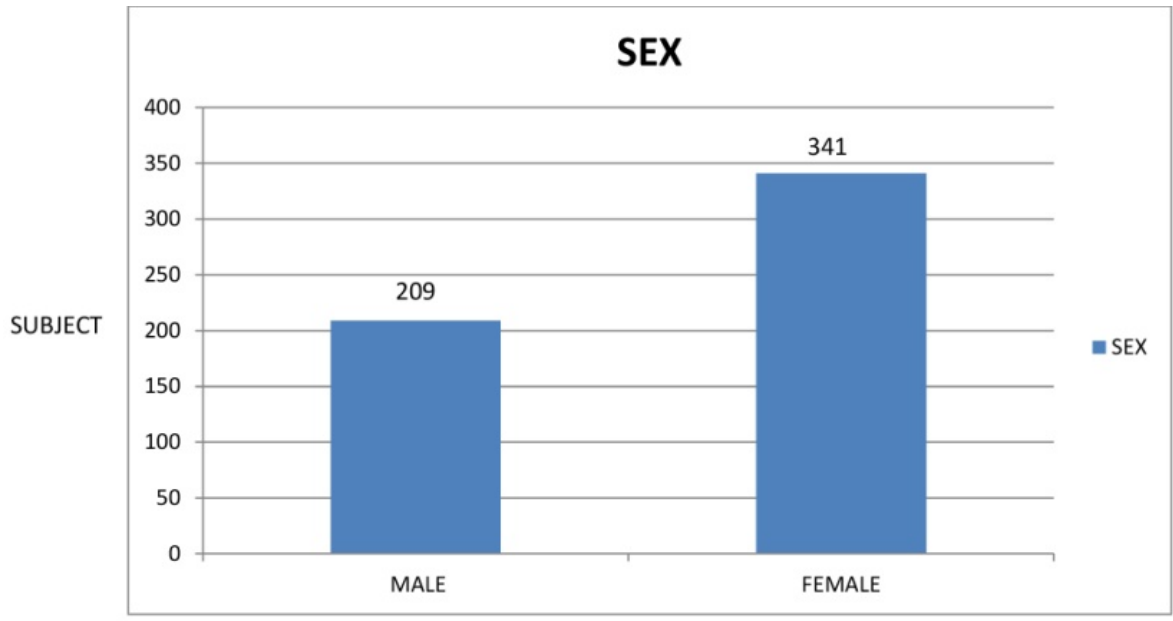

Fig. 1: Graph I.

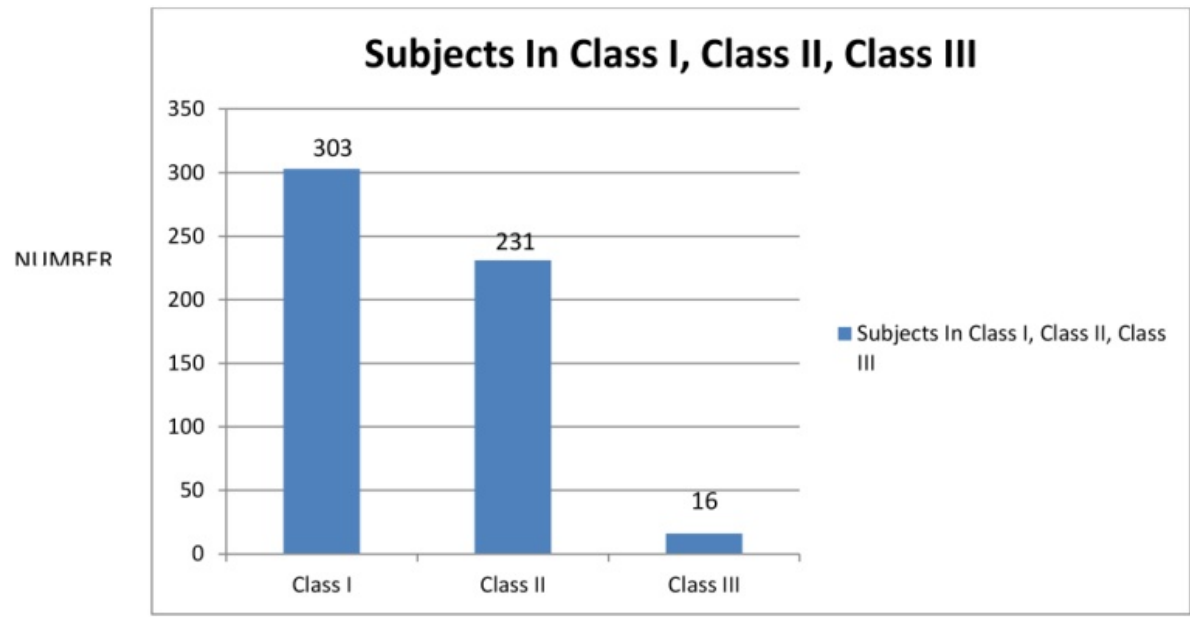

Fig. 2: Graph II. 


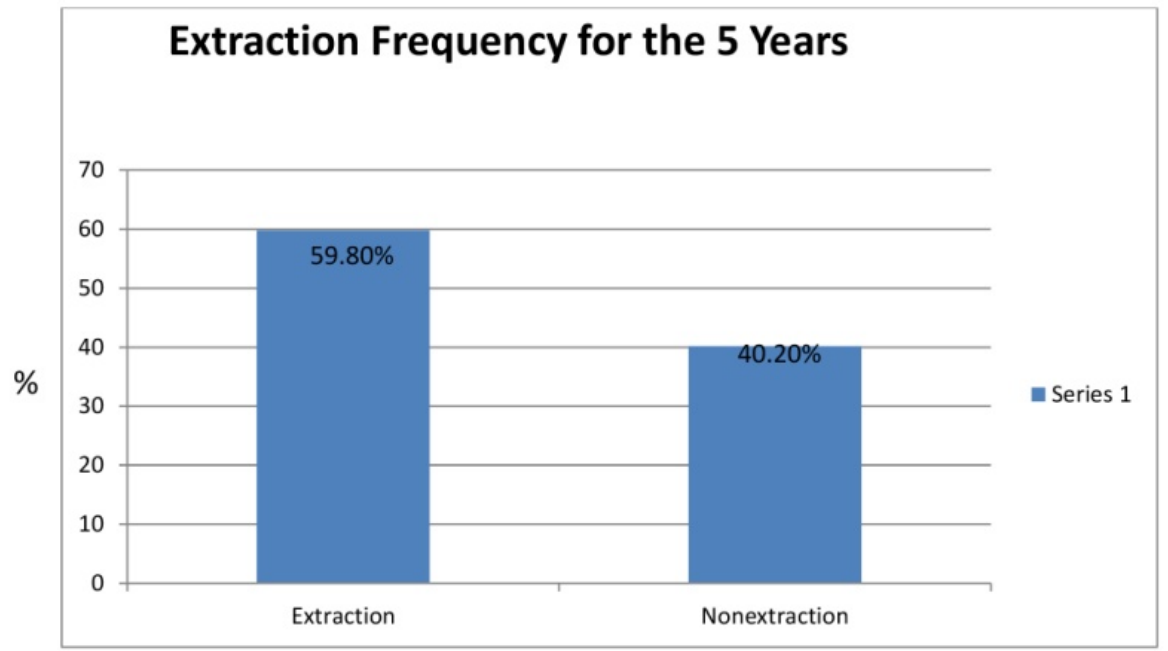

Fig. 3: Graph III.

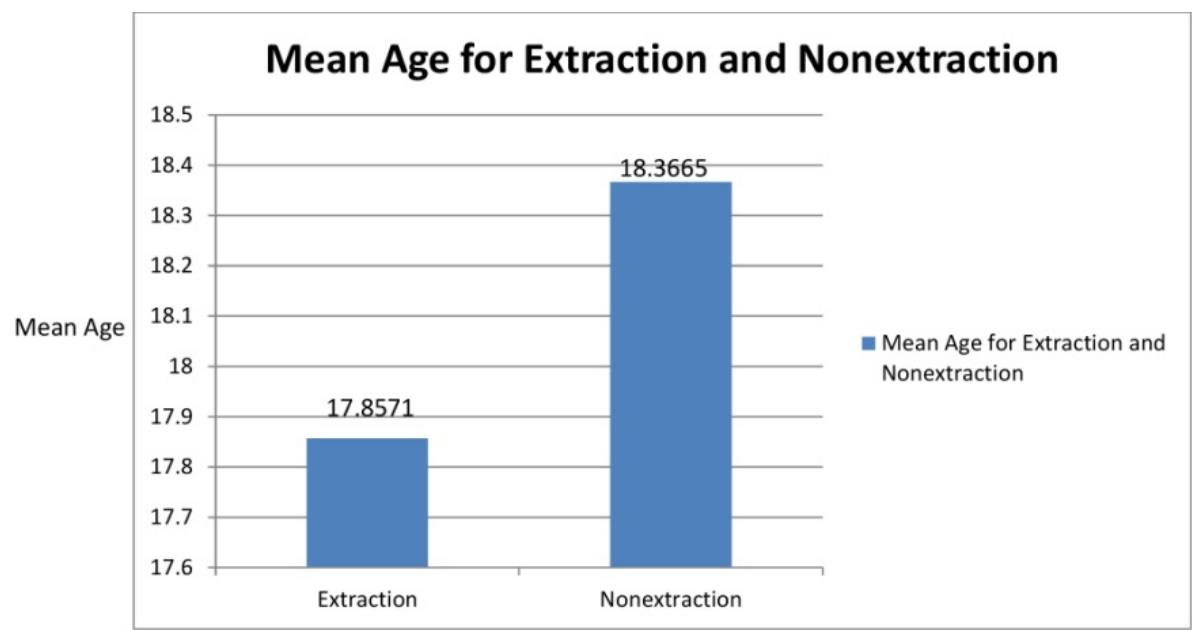

Fig. 4: Graph IV.

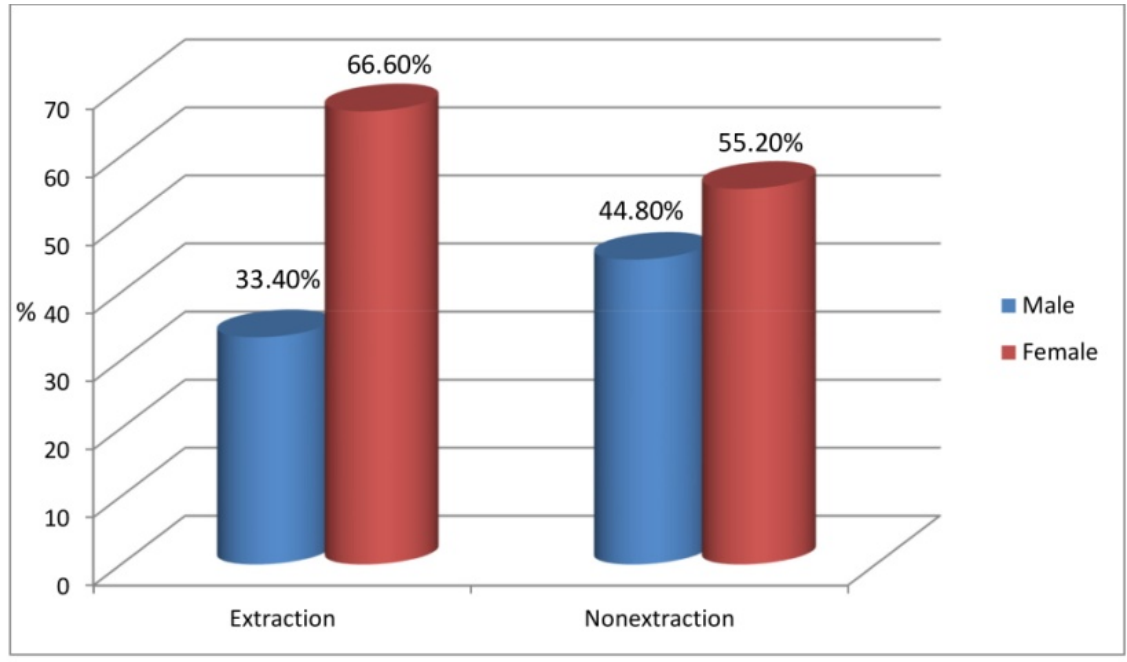

Extraction vs Nonextraction among Sex

Fig. 5: Graph V. 


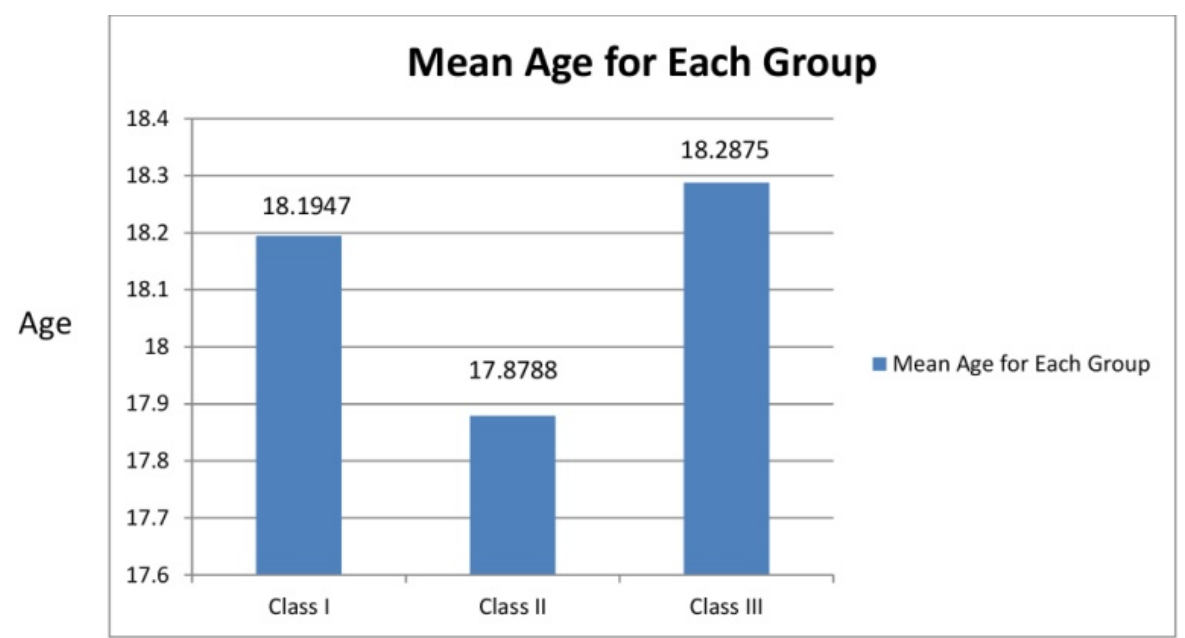

Fig. 6: Graph VI.

the entire sample, in males the frequency of extraction for the entire sample was $33.4 \%$ where as in females the frequency of extraction was $66.6 \%$ (Fig. 5). In the non extraction group, the percentage of males was $44.8 \%$, where as in females the percentage of non extraction was $55.5 \%$ (Fig. 5).

The mean age of class I, class II, and class III subjects were 18, 19, 17.87 and 18.19 years respectively (Fig. 6). Figure 7 , the frequency of extraction in each group was from 303 class I subjects 181 subjects (59.73\%) underwent extractions and $122(40.26 \%)$ underwent the non extraction treatment. Among these 52 (28.7\%) males and $129(71.3 \%)$ females underwent extraction and $55(45.1 \%)$ males and $67(54.9 \%)$ females underwent the non extraction protocol (Table 2) (Fig. 8).

Figure 7, from 231 class II subjects, 142 (61.47\%) subjects underwent extraction and 89 (38.52\%) subjects underwent the non- extraction treatment (Fig. 9) among these $56(39.4 \%)$ males and 86 (60.6\%) females underwent extraction and $38(42.7 \%)$ males and 51 $(57.3 \%)$ females underwent the non- extraction protocol. (Table 3) from 16 class III subjects, 6 (37.5\%) subjects underwent extraction and $10(62.5 \%)$ subjects underwent non- extraction (Fig. 10) among these 2 (33.3\%) males and $4(66.7 \%)$ females underwent extraction and $6(60 \%)$ males and $4(40 \%)$ females underwent nonextraction (Table 4).

\section{Discussion}

Clinicians and researchers are interested in determining the basis of the clinical judgments that are made during the diagnosis and treatment planning of orthodontic ca-

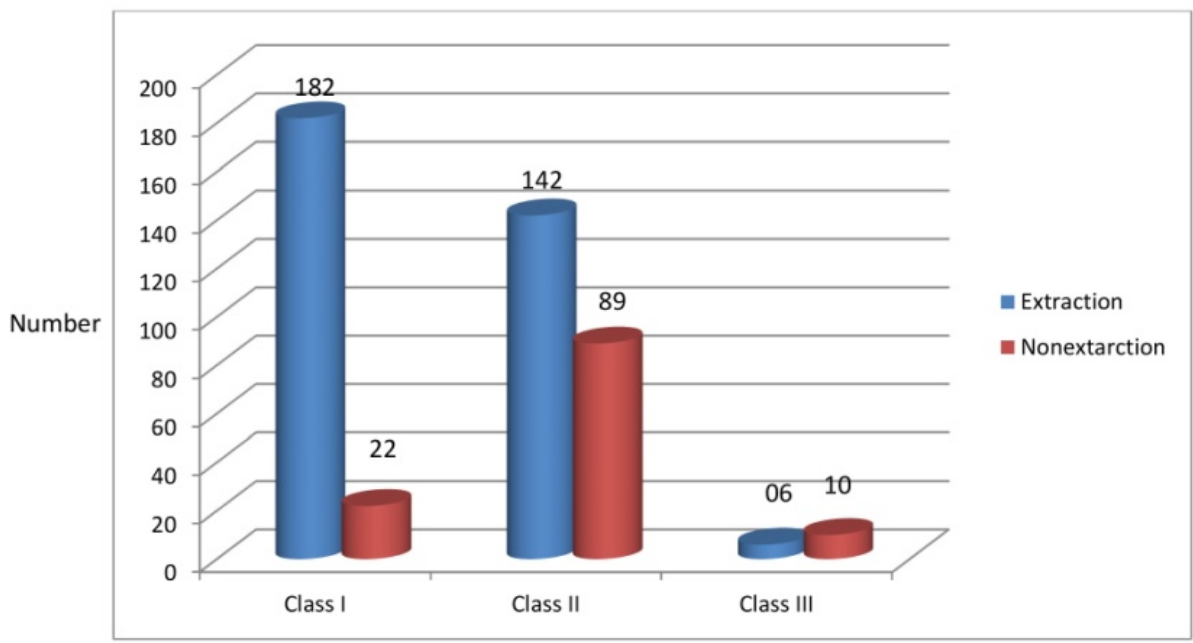

Fig. 7: Graph VII.

Number of Subjects in Each Group 
Table 2: Class I and sex extraction status. extraction status sex cross tabulation.

\begin{tabular}{|l|c|c|c|c|c|}
\hline & & & Sex & & Total \\
\hline & & & Extraction & Nonextraction & 181 \\
\hline EXTNSTS & 1.00 & Count & 52 & 129 & $100.0 \%$ \\
\hline & & $\%$ Within EXTNSTS & $28.7 \%$ & $71.3 \%$ & $59.7 \%$ \\
\hline & & $\%$ Within SEX & $48.6 \%$ & $65.8 \%$ & 122 \\
\hline & 2.00 & Count & 55 & 67 & $100.0 \%$ \\
\hline & & $\%$ Within EXTNSTS & $45.1 \%$ & $54.9 \%$ & 40.3 \\
\hline Total & & $\%$ Within SEX & $51.4 \%$ & $34.2 \%$ & 303 \\
\hline Total & & Count & 107 & 196 & $100.0 \%$ \\
\hline
\end{tabular}

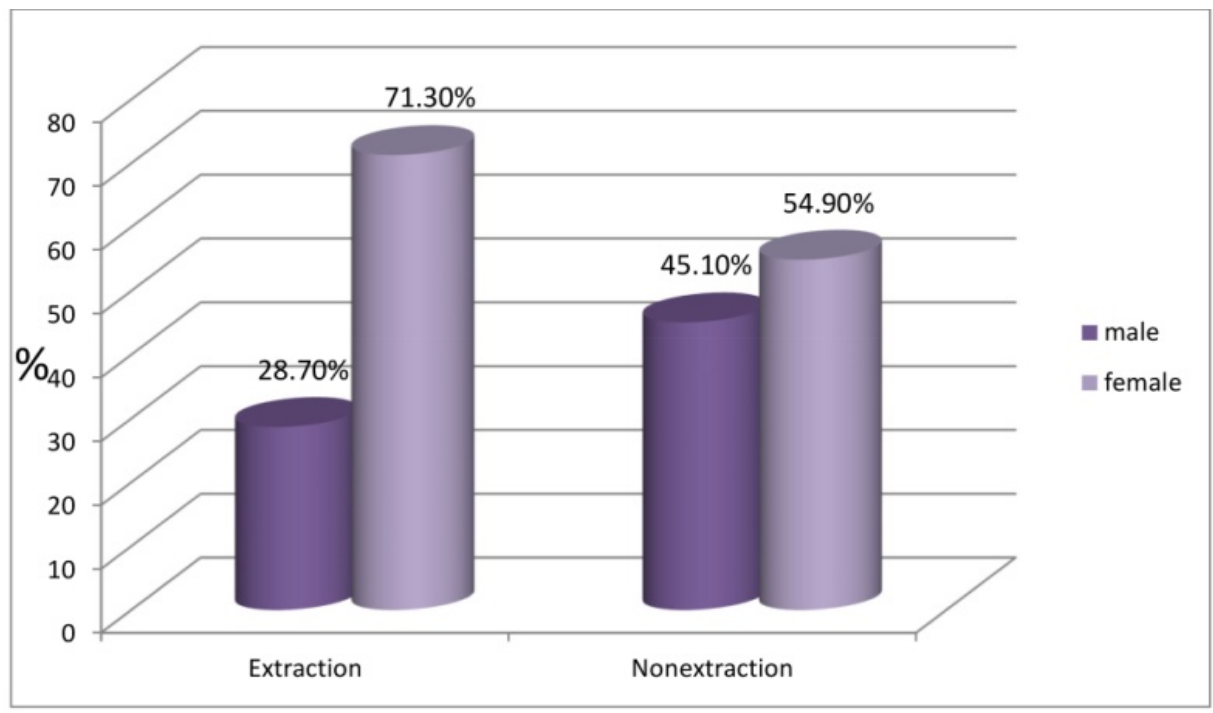

Class I Group Extraction Status

Fig. 8: Graph VIII.

ses. Although a number of these judgments are subjective and systematic, hence quantifiable.

In this retrospective study with a sample size of 550, it was found that $329(59.8 \%)$ of the subjects underwent extraction and $221(40.2 \%)$ subjects underwent nonextraction (Fig. 1). This was because majority of the patients came to the orthodontic clinic with complaint of protrusive lips, proclined upper and lower incisors and crowding of either the upper or lower teeth, which could not be corrected solely by nonextraction as it would worsen the profile. Also the patients who had a mildly convex profile prefer a straight profile which was related to their ethnic background as most of the patients were Indians. This was in concordance with a study done by Siddhartha Dhar et al (7).

Among the total of 550 subjects 341 were females $(62 \%)$ and the remaining 209 were males (38\%). This is due to the fact that females were more concerned about their appearance and hence motivated for the orthodontic treatment.

The mean age in extraction group was $17.85+/-4.18$ years and the mean age for nonextraction was $18.36+/-$ 4.89 years. So age was not a statistically significant factor for the frequency of extraction in the entire sample (Fig. 3). Among the total 329 subjects who had undergone extraction, $66.6 \%$ were females and the rest $33.4 \%$ were males. This is because females preferred a straight profile while most of the males who had a mildly convex profile were satisfied with their profile (Fig. 4).

Based on Angle's classification of malocclusion the subjects were divided into Class I, Class II and Class III groups (Fig. 2). Among the Class I subjects (303), 181 subjects underwent extraction and remaining 122 were treated with the non extraction protocol. The most com- 


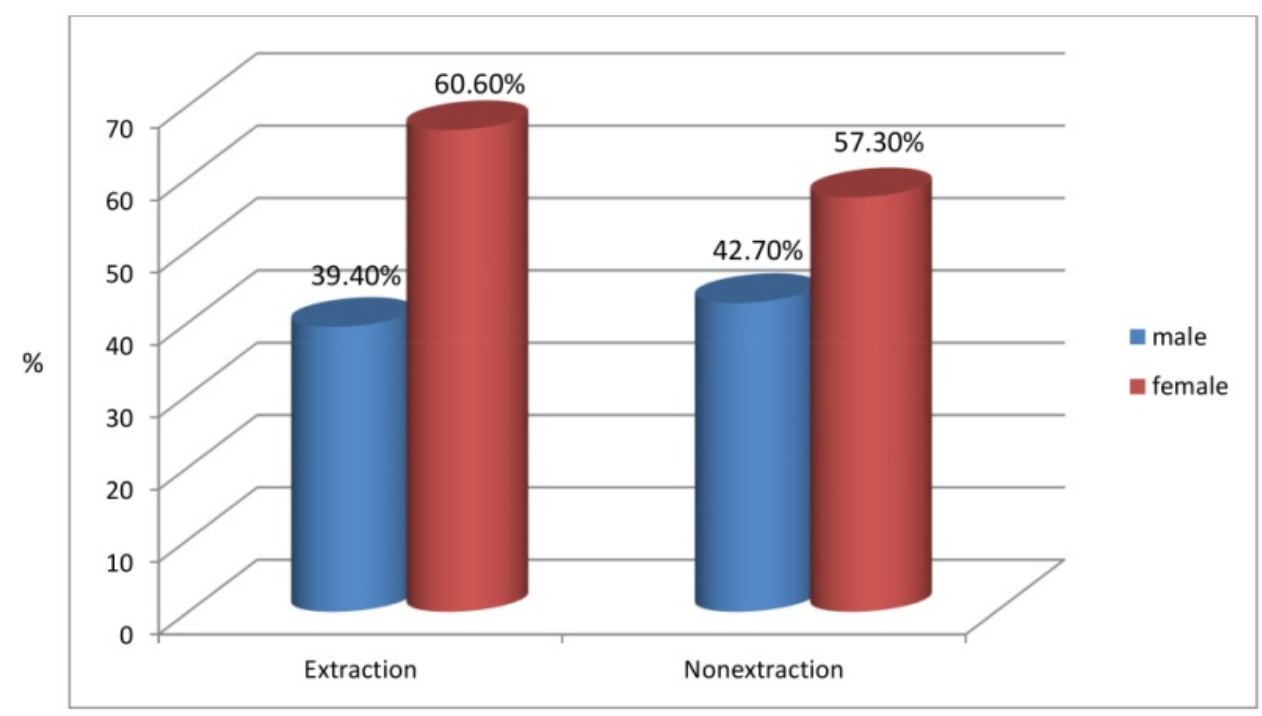

Fig. 9: Graph IX.

CLASS II GROUP EXTRACTION STASTUS

Table 3: Class II extraction status. extnsts: sex cross tabulation.

\begin{tabular}{|l|c|c|c|c|}
\hline & & \multicolumn{2}{|c|}{ SEX } & Total \\
\hline & & male & female & \\
\hline Extraction & Count & 56 & 86 & 142 \\
\hline & $\%$ Within EXTNSTS & $39.4 \%$ & $60.6 \%$ & $100.0 \%$ \\
\hline & $\%$ Within SEX & $59.6 \%$ & $62.8 \%$ & 61.5 \\
\hline nonextraction & Count & 38 & 51 & 89 \\
\hline & $\%$ Within EXTNSTS & $42.7 \%$ & $57.3 \%$ & $100.0 \%$ \\
\hline & \% Within SEX & $40.4 \%$ & $37.2 \%$ & 38.5 \\
\hline Total & Count & 94 & 137 & 231 \\
\hline & $\%$ Within EXTNSTS & $40.7 \%$ & $59.3 \%$ & $100.0 \%$ \\
\hline & \% Within SEX & $100.0 \%$ & $100.0 \%$ & $100.0 \%$ \\
\hline
\end{tabular}

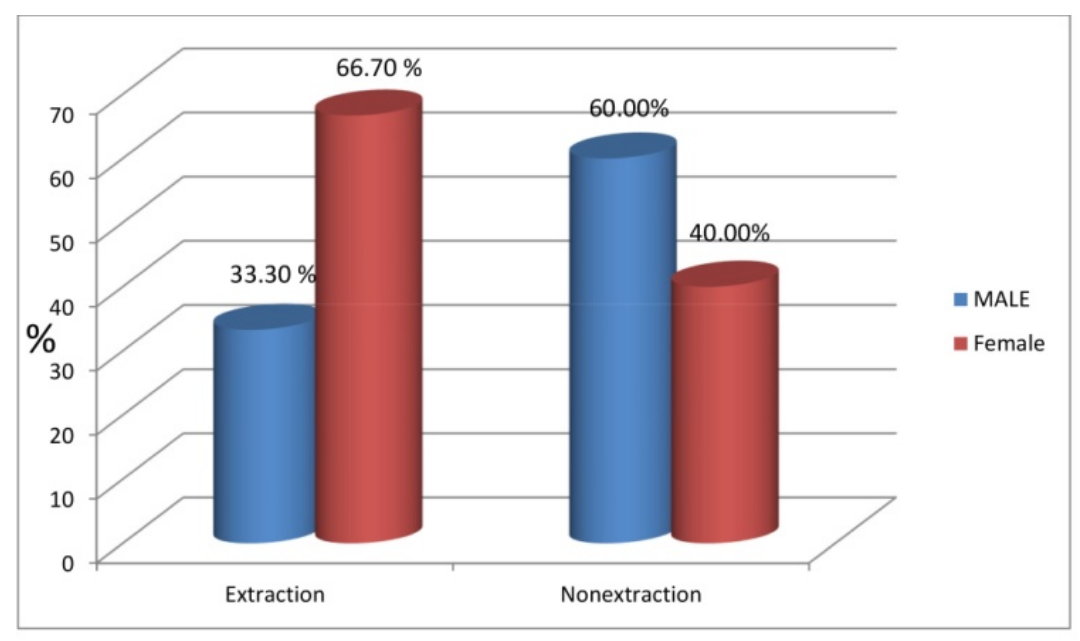

Fig. 10: Graph X.

\section{Class III Group Extraction status}


Table 4: Class III extraction status. extnsts: sex cross tabulation.

\begin{tabular}{|l|c|c|c|c|}
\hline & & \multicolumn{2}{|c|}{ SEX } & Total \\
\hline & & Male & Female & \\
\hline Extraction & Count & 2 & 4 & 6 \\
\hline & $\%$ Within EXTNSTS & $33.3 \%$ & $66.7 \%$ & $100.0 \%$ \\
\hline & $\%$ Within SEX & $25.0 \%$ & $50.0 \%$ & $37.5 \%$ \\
\hline Nonextraction & Count & 6 & 4 & 10 \\
\hline & $\%$ Within EXTNSTS & $60.0 \%$ & $40.0 \%$ & $100.0 \%$ \\
\hline & $\%$ Within SEX & $75.0 \%$ & $50.0 \%$ & $62.5 \%$ \\
\hline Total & Count & 8 & 8 & 16 \\
\hline & $\%$ Within EXTNSTS & $50.0 \%$ & $50.0 \%$ & $100 \%$ \\
\hline & $\%$ Within SEX & $100.0 \%$ & $100.0 \%$ & $100.0 \%$ \\
\hline
\end{tabular}

mon malocclusion for these patients was crowding and bimaxillary protrusion. Both of these are associated with tooth size arch length discrepancy so these patients were mostly treated by means of extraction of the four first premolars since intentional widening or expansion of the dental arches often is avoided especially when standard edgewise appliance are used, because of the known tendency to relapse according to McNamara.

The present study among the 181 class 1 extraction subgroup (Fig. 8) majority of the females 129 (71.3\%) were South Indians with bimaxillary protrusion having a convex profile including few Chinese females. Whereas the Class I non extraction subgroup involved Indian males and females with mildly convex teeth and less crowding of teeth compared to the Class I extraction subgroup. So it is necessary to take into consideration the patients' ethnic background, skeletal, dental and physiological age, the function and malformation of teeth and jaws and the soft tissue configuration of the face. Thought the mean age of the Class I extraction group 18.81 years, the tooth size arch length discrepancy $-2.33 \mathrm{~mm}$ and the proclination of lower incisor to NB of $9.5 \mathrm{~mm}$ in the Class I extraction group was very highly significant $(p=0.001)$ (Table 2) compared to the non extraction group with tooth size arch length discrepancy of $0.5 \mathrm{~mm}$ and lower incisor to NB of $6.86 \mathrm{~mm}$. Also the mean age of both the Class I extraction and non extraction subgroup was beyond the adolescent growth spurt for mandibular growth to take place.

\section{Conclusions}

1. The frequencies of extraction for 5 years from 2007 to 2016 in the S.D.M. College of Dental Sciences and Hospital of orthodontic department from a sample size of 550 subjects was $59.8 \%$ of 329 subjects who underwent extraction and $40.2 \%$ with 221 subjects who underwent nonextraction line of treatment comprising higher frequency of extraction in general.

2. The frequency of extraction for class I malocclusion comprising 303 subjects were [59.73\%] and 181 subjects who underwent extraction and 122 [40.26\%] who underwent nonextraction line of treatment. The frequency of extraction for class II malocclusion comprising 231 subjects were [61.47\%] and 142 subjects who underwent extraction and 89 [38.52\%] who underwent nonextraction line of treatment. The frequency of extraction for class III malocclusion comprising 16 subjects [37.5\%] and 6 subjects who underwent extraction and 10 [62.5\%] who underwent nonextraction line of treatment shows that among all the groups, Class I malocclusion shows higher frequency of extraction.

3 . The mean age of males was extraction was $17.85+/-$ 4.18 and the mean age of females was $18.36+/ 44.89$ which was not statistically significant [males $p=.192$, females $p=.206]$. (Fig. 4) showing extraction frequency is more in late adolescent period.

4. While relating sex with the frequency of extraction for the entire sample, in males the frequency of extraction was $33.4 \%$ where as in females the frequency of extraction was $66.6 \%$ (Fig. 5). In the non extraction group, the percentage of males was $44.8 \%$, where as in females the percentage of nonextraction was $55.2 \%$ (Fig. 5) shows that there was higher frequency of extraction in females.

\section{References}

1. Bishara SE, Cummins DM, Jacobsen JR. The morphologic basis for the extraction decision in class 2 Division 1 Malocclusion: A comparative study. Am J Orthod. 1995;107:129-135.

2. William R Profit. Forty year review of extraction frequencies at a university clinic. Angle Orthod. 1994;64:407-414.

3. Charles 2. Tweed. Clinical Orthodontics Vol 1, St Louis Mosby. 1966;1:56-60.

4. Bowman SJ, Johnston LE. The esthetic impact of extraction and non extraction treatments on Caucasian patients. Am J Orthod. 2000;70:3-10.

5. Weijntraub JA, Vig PS, Brown C, Kowalski CJ. The Prevalence of Orthodontic Extractions. Am J Orthod. 1989;96:462-466.

6. Peck S, Peck H. Frequency of tooth extraction in orthodontic treatment. Am J Orthod. 1979;76:491-496.

7. Dhar S, Ghandhi S. The impact of ethnic background and environment on female facial profile preferences among young layman of north Indian Origin: A three nation study. Am J Orthod. 2006;129:830836. 
8. Farrar JN. Irregularities of the teeth and their corrections, vol 1, p, 658, New York City, 1888.

9. Pierce CN. Regulating the Natural Denture. Dent Cosmos. 1859;6:113-115.

10. Angle EH. Treatment of Malocclusion of teeth, 7th Ed, Philadelphia, SS White Manufacturing Co. 1970.125-32.

11. Dewel B. The Case Drewrey - Cryer extraction debate: A Commentary. Am J Orthod. 1964;50:862-5.

12. Tweed $\mathrm{CH}$. Indications for the extraction of teeth in orthodontic procedure. Am J Orthod. 1944;20:405-428.

13. Begg PR. Stone age man's dentition. Am J Orthod. 1954;40:298312.

14. Ceylan I, Yavuz I, Arslan F. The Effect of Overjet on Dentoalveolar Compensation. Eur J Orthod. 2003;25:325-330.

15. Abu Alhaija ES, Al-Khateeb SN. Attractiveness ratings of anterior open bites and reverse ovejets using the aesthetic component of the index of Orthodontic Treatment Needs. Eur J Orthod. 2005;27:134-9. 16. Douglas Squire, Al M Best, Steven J, Lindauer and Daniel M Laskin. Determining the limits of Orthodontic Treatment of overbite, overjet and transverse discrepancy: A Pilot study. Am J Orthod. 2006;129:804-8

17. Poopwich K, Nebbe B, Heo G Glover KE, Major PW. Predictors for Class 2 treatment Duration. Am J Orthod. 2005;127:293-300.

18. Turkkahraman H, Sayinmo. Relationship between Mandibular anterior Crowding and lateral Dentofacial Morphology in the early mixed dentition. Angle Orthod. 2004;74:759-64.

19. Baydas B, Yavuz I, Dagsuyu IM, Bolukbasi B, Ceylan I. An investigation of maxillary and mandibular morphology in different overjet groups. Aust Ortho J. 2004;20:11-8.

20. Ceylan I, Baydas B, Bolabkbasib. Longitudinal Cephalometric changes in incisor position, overjet, overbite between $10-14$ years of age. Angle Orthod. 2002;72:246-250.

\section{Conflict of interest}

The authors have declared that no conflict of interest exist. 